\title{
Activity of Melatonin Against Gastric Cancer Growth in a Chick Embryo Tumor Xenograft Model
}

\author{
Rixiong Wang ${ }^{1,2}$ \\ Hui Liu ${ }^{3,4}$ \\ Jun Song ${ }^{3,4}$ \\ Qing $\mathrm{Wu}^{1,2}$
}

'Department of Oncology, the First Affiliated Hospital of Fujian Medical University, Fuzhou City, People's Republic of China; ${ }^{2}$ Fujian Key Laboratory of Precision Medicine for Cancer, the First Affiliated Hospital of Fujian Medical University, Fuzhou City, People's Republic of China; ${ }^{3}$ Department of Human Anatomy, Histology and Embryology, Fujian Medical University, Fuzhou City, People's Republic of China; ${ }^{4} \mathrm{Key}$ Laboratory of the Ministry of Education for Gastrointestinal Cancer, Fujian Medical University, Fuzhou City, People's Republic of China
Correspondence: Rixiong Wang Email 13960758357@fjmu.edu.cn
Purpose: Previous studies have shown the antitumor activity of melatonin against a wide range of human cancers; however, the impact of melatonin on gastric cancer growth remains to be illustrated. This study aimed to investigate the activity of melatonin against gastric cancer growth in a chick embryo tumor xenograft model and explore the possible mechanisms.

Materials and Methods: The growth of gastric cancer SGC-7901 cells was measured using MTT assay, and a chick embryo tumor xenograft model was generated to observe the effect of melatonin on gastric cancer growth in vivo. In addition, the VEGF and angiogenin secretion was measured in the supernatant of chick embryo tumor xenograft models with ELISA.

Results: MLT treatment inhibited the growth of SGC-7901 cells at a concentration-dependent manner, and treatment with MLT at $1 \mathrm{mM}$ was found to markedly reduce the volume and weight of tumors bearing the allantois of chicken embryos. ELISA showed that MLT at concentrations of $0.0041,0.012,0.037$ and 0.11 had no remarkable impact on VEGF and angiopoietin secretion, while MLT at $1 \mathrm{mM}$ significantly suppressed VEGF and angiopoietin production in chick embryo tumor xenograft models with SGC-7901 cells $(P=0.023)$.

Conclusion: Our data demonstrate that MLT inhibits gastric cancer growth in vitro at a concentration-dependent manner, and suppresses angiogenesis of the chick embryo tumor xenograft model with SGC-7901 cells through inhibiting VEGF and angiogenin secretion. Further studies are needed to investigate the therapeutic potential of MLT for gastric cancer as compared to drugs clinically approved.

Keywords: gastric cancer, melatonin, growth, angiogenesis, vascular endothelial growth factor, angiogenin, chick embryo tumor xenograft model

\section{Introduction}

Gastric cancer, also known as stomach cancer, is the sixth most frequent malignancy and the fourth leading cause of death in the world. ${ }^{1}$ Globally, there were 1.09 million new cases diagnosed with gastric cancer, and 769,000 deaths occurred due to gastric cancer. ${ }^{2}$ Gastric cancer was estimated to cause 19.1 million (95\% uncertainty interval, 18.7-19.6 million) disability-adjusted life-years (DALYs) in 2017, with 98\% from years of life lost (YLLs) and 2\% from years lived with disability (YLDs), and there were 1 in 33 men and 1 in 78 women that developed gastric cancer over a lifetime. ${ }^{3}$ The clinical prognosis of gastric cancer predominantly depends on stage. ${ }^{4}$ The 5 -year survival rate of early-stage gastric cancer is approximately $90 \%{ }^{5}$ however, this malignancy is frequently diagnosed at a late stage because of lack of specific clinical symptoms at early stage, leading to unfavorable prognosis and low survival rate. ${ }^{6}$ Improving the survival remains a major clinical challenge in gastric cancer. ${ }^{7-11}$ 
Angiogenesis, a biological process that is critical to both physiological and pathological processes such as wound healing and tumor growth, is the formation of new blood vessels from pre-existing microvasculature, and is regulated by an opposing balance of angiogenic and angiostatic factors. ${ }^{12}$ Angiogenesis not only provides energy and oxygen supplies for cancer cells, but also directs cancer cells into blood circulation and their migration into distant locations. ${ }^{13}$ Angiogenesis has shown a critical role in tumor growth, metastasis and prognosis, which is strongly associated with survival in cancer patients, ${ }^{14}$ and inhibition of tumor cell growth and metastasis may prevent tumor angiogenesis. ${ }^{15}$ Blockade of angiogenesis is therefore accepted as a novel treatment for human cancers. ${ }^{16}$

Melatonin (MLT), a methoxyindole that is primarily synthesized and secreted by the pineal gland, ${ }^{17}$ has been found to exhibit a wide range of pharmacological actions including detoxification of free radicals and antioxidant actions, bone formation and protection, reproduction, and cardiovascular, immune or body mass regulation, and brain or gastrointestinal protection, ${ }^{18-20}$ as well as suppression of cancer development, progression and metastasis. ${ }^{21}$ Our previous studies found that MLT inhibited gastric cancer growth in vivo and in vitro and exhibited an anti-angiogenic and antitumor activity against gastric cancer by targeting HIF-1 $\alpha$, which stabilizes the tumor microenvironment and stimulates tumor angiogenesis. ${ }^{22-25}$ The present study aimed to examine the impact of MLT on angiogenesis of gastric cancer in a chick embryo tumor xenograft model.

\section{Methods}

\section{Ethical Statement}

This study was approved by the Animal Experimental Ethical Inspection Committee of Fujian Medical University (approval number: FJMU IACUC 2018-003). All animal experiments were performed strictly according to the international and national laws, regulations and guidelines for the management and care of laboratory animals.

\section{Cell Line and Culture}

Human gastric cancer SGC-7901 cell line was purchased from Shanghai Institute for Biological Science of the Chinese Academy of Sciences (Shanghai, China). Cells were cultured in RPMI-1640 medium (Invitrogen; Carlsbad, CA, USA) supplemented with $10 \%$ fetal bovine serum (FBS; Invitrogen; Carlsbad, CA, USA) and maintained at $37^{\circ} \mathrm{C}$ containing $5 \% \mathrm{CO}_{2}$.

\section{Cell Proliferation and Viability Assay}

SGC-7901 cells were seeded onto 96-well plates (Corning, Inc.; Corning, NY, USA) at a density of 5000 cells per well. Cells were exposed to MLT at concentrations of 0.0014 , $0.0041,0.012,0.037,0.11,0.33,1$ and $3 \mathrm{mM}$ for 24 hours, while cells treated with cisplatin and RPMI-1640 medium alone served as positive and negative controls. Cell proliferation was measured using MTT assay (Invitrogen; Carlsbad, CA, USA) following the manufacturer's guidelines. All measurements were repeated in triplicate.

\section{Chick Embryo Chorioallantoic Membrane Assay}

Pathogen-free fertilized white leghorn chicken (Gallus gallus domesticus) eggs were obtained from Henry Stewart Co., Ltd. (UK), and chicken embryos, which hatched for 5 days, were maintained at $37^{\circ} \mathrm{C}$ containing $60 \% \mathrm{CO}_{2}$. The air chambers were opened under sterile conditions, and the crust endomembrane was exposed and implanted on the chorioallantoic membrane at the embryonic development day in the flank with $2 \times 10^{6}$ SGC-7901 cells.

Chicken embryos with SGC-7901 cells were randomly assigned into two groups, of 10 chick embryos in each group. Slips containing normal PBS (control group) and the PBS containing $1 \mathrm{mM}$ MLT (experimental group) were stuck to the chicken embryonic allantois. The holes were enclosed with paraffin and cover slides. The long $(L)$ and short (W) axes of the tumors were measured with a caliper, and the tumor size $(\mathrm{V})$ was calculated using the following formula: $\mathrm{V}=4 / 3 \pi \times \mathrm{L} / 2 \times(\mathrm{W} / 2){ }^{2}$ Blood vessels were classified into three types: class 1 blood vessels were defined as bole vessels, class 2 blood vessels as branches on the bole vessels, and class 3 blood vessels as branches following class 2 blood vessels. The number of blood vessels at each class was blindly counted by three investigators. Tumors were collected at the end of the study and tumor weight was blindly measured by three investigators.

\section{Enzyme-Linked Immunosorbent Assay (ELISA)}

Chick embryo tumor xenograft models with SGC-7901 cells were hatched for 10 days, and the VEGF and angiopoietin 
levels were measured in the supernatants secreted by the cultured cancer cells using ELISA. Briefly, the cultured supernatants were collected and centrifuged at 12,000 rpm at $4{ }^{\circ} \mathrm{C}$ for $15 \mathrm{~min}$, and the secretion of VEGF and angiopoietin was detected using VEGF-ELISA (R \& D Systems, Inc.; Minneapolis, MN, USA) and ANG-ELISA kits (R \& D Systems, Inc.; Minneapolis, MN, USA) following the manufacturer's instructions. The values of optical density were measured at a wave length of $450 \mathrm{~nm}$, and all measurements were repeated in triplicate.

\section{Statistical Analysis}

All measurement data were presented as the mean \pm standard deviation (SD), and all statistical analyses were performed using the statistical software SPSS version 16.0 (SPSS, Inc.; Chicago, IL, USA). Differences of means between groups were tested for statistical significance with Student's $t$ test, and one-way analysis of variance (ANOVA) was used to compare differences in repeated measures across experiments. A $P$ value of $<0.05$ was considered statistically significant.

\section{Results}

\section{MLT Suppresses Gastric Cancer Cell Growth}

To examine the effects of MLT treatment on SGC-7901 cells, the cell growth inhibition in response to MLT treatment was examined. MLT treatment resulted in a significant reduction in the number of SGC-7901 cells, and MLT treatment was found to suppress the growth of SGC-7901 cells at a concentration-dependent manner (Figure 1). As a positive control, the inhibition of cisplatin on the growth of SGC7091 cells was also observed (Figure S1).

\section{MLT Suppresses Gastric Cancer Growth in a Chick Embryo Tumor Xenograft Model}

Since the antitumor activity of MLT has been extensively described previously [21], the present study sought to determine the effective concentration of MLT that was required to inhibit the growth of the chick embryo tumor xenograft model with SGC-7901 cells. Treatment with MLT at $1 \mathrm{mM}$ was found to markedly reduce the volume and weight of tumors bearing the allantois of chicken embryos (Figure 2). Our data further confirmed the activity of MLT against gastric cancer.

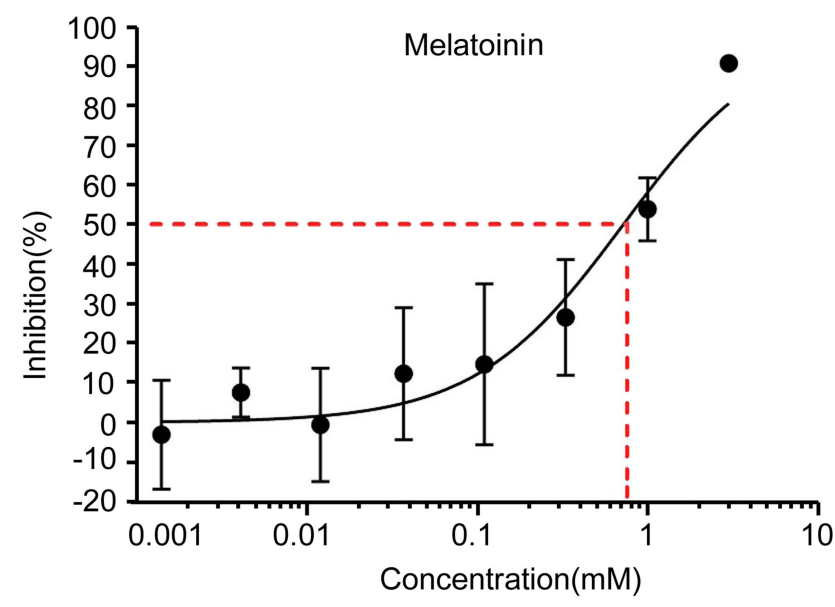

Figure I Effects of melatonin on the growth of human gastric cancer SGC-790 cells. Cells were exposed to MLT at concentrations of $0.0014,0.0041,0.012,0.037$, $0.1 \mathrm{I}, 0.33$, I and $3 \mathrm{mM}$, while cells treated with RPMI- 1640 medium alone served as the negative control. MTT was employed to determine cell proliferation. The inhibition (\%) was calculated as: cell growth upon MLT treatment/cell growth upon negative control treatment. Data are expressed as the mean \pm SD from three independent experiments.

\section{MLT Suppresses VEGF and Angiopoietin Secretion in a Chick Embryo Tumor Xenograft Model}

To unravel the potential mechanism underlying the inhibition of MLT on gastric cancer growth, we investigated the impairment of angiogenesis in chick embryo tumor xenograft models following exposure to MLT by measuring VEGF and angiopoietin levels. ELISA showed that low concentrations of MLT had no remarkable impact on VEGF and angiopoietin secretion, while MLT at $1 \mathrm{mM}$ significantly suppressed VEGF and angiopoietin production in chick embryo tumor xenograft models with SGC-7901 cells $(P=0.023)$, which is consistent with the growth-inhibitory effects (Figure 3 ).

\section{Discussion}

Previous studies have demonstrated that MLT is active to inhibit the proliferation of multiple human cancers, including gastric cancer, ${ }^{17-21}$ and our previous studies have confirmed the inhibition of MLT on gastric cancer proliferation in vivo and in vitro through suppressing HIF-1a accumulation and VEGF generation via the nuclear receptor RZR/ROR $\gamma^{22-25}$ The antitumor effect of melatonin is mainly attributed to its powerful immune-regulatory functions, anti-proliferation effects, anti-oxidative and antiangiogenetic actions, which is one of the most important biological bases for tumor growth and development. ${ }^{26-28}$

In this study, we tested the activity of MLT at concentrations of $0.0041,0.012,0.037,0.11,0.33,1$ and 3 
A

PBS D0

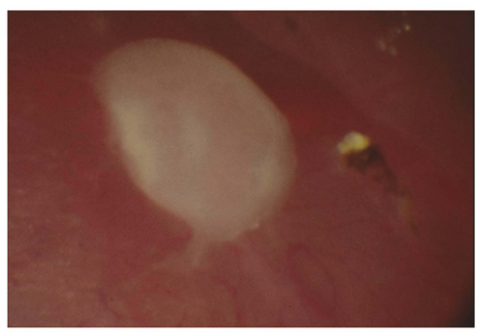

Melatonin D0

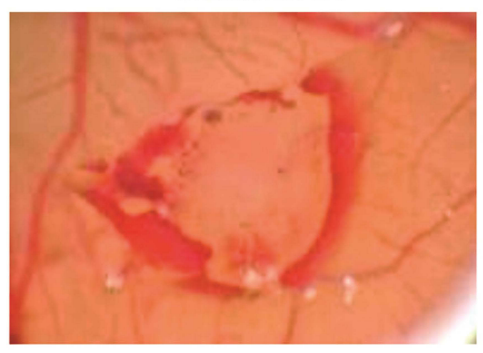

PBS D5

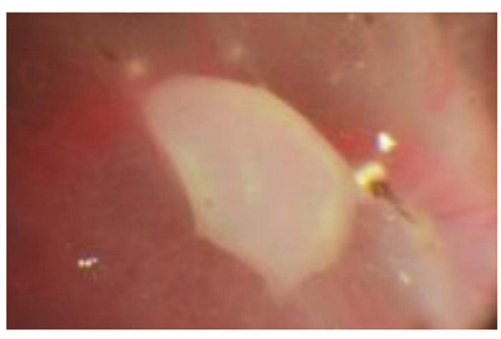

Melatonin D5

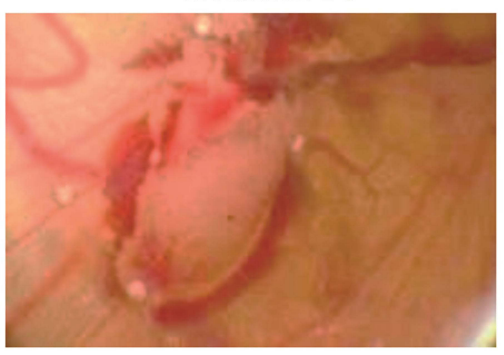

PBS D9

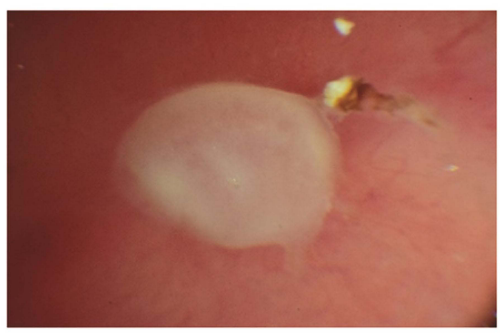

Melatonin D9

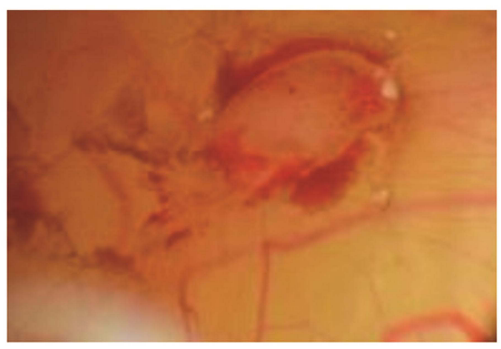

B

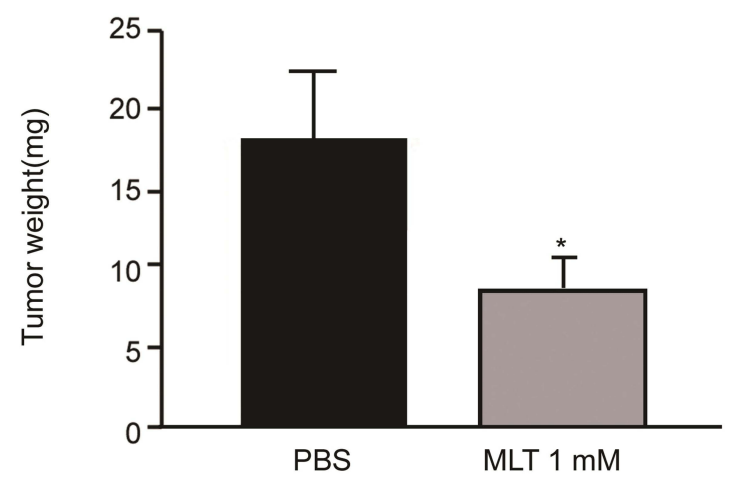

D

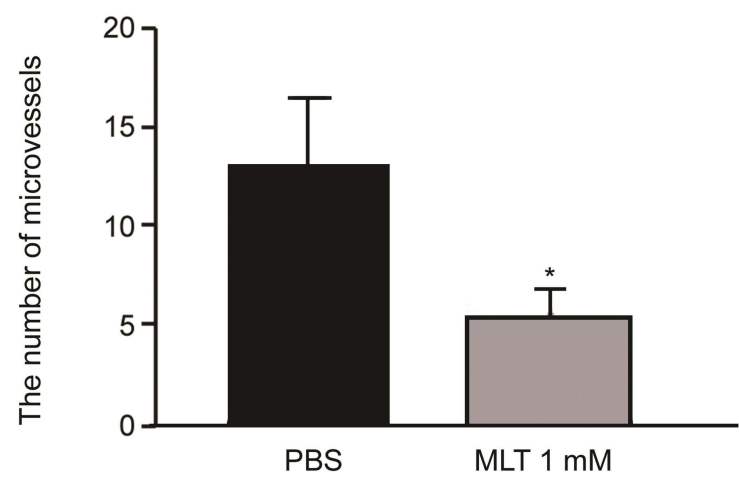

C

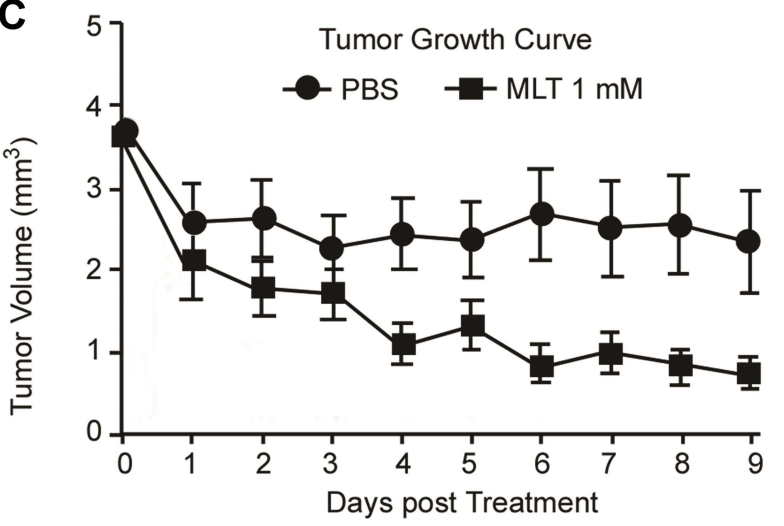

Figure 2 Melatonin inhibits microvessel formation and tumor proliferation of the chick embryo tumor xenograft model. (A) Changes of chick embryo tumor xenografts during administration with melatonin; (B) Comparison of tumor weight; (C) Comparison of tumor volume; (D) Comparison of allantois of chick embryos. Data are presented as the mean \pm standard SD. $* P<0.05$ vs controls.

Abbreviation: MLT, melatonin.

mM against human gastric cancer SGC-7901 cells. MTT assay showed that MLT at concentrations of 0.0041, $0.012,0.037,0.11$ and $0.33 \mathrm{mM}$ had no remarkable inhibition on SGC-7901 cells, while MLT at 1 and 3 mM significantly suppressed SGC-7901 cell growth. In addition, our data showed that MLT suppressed the 

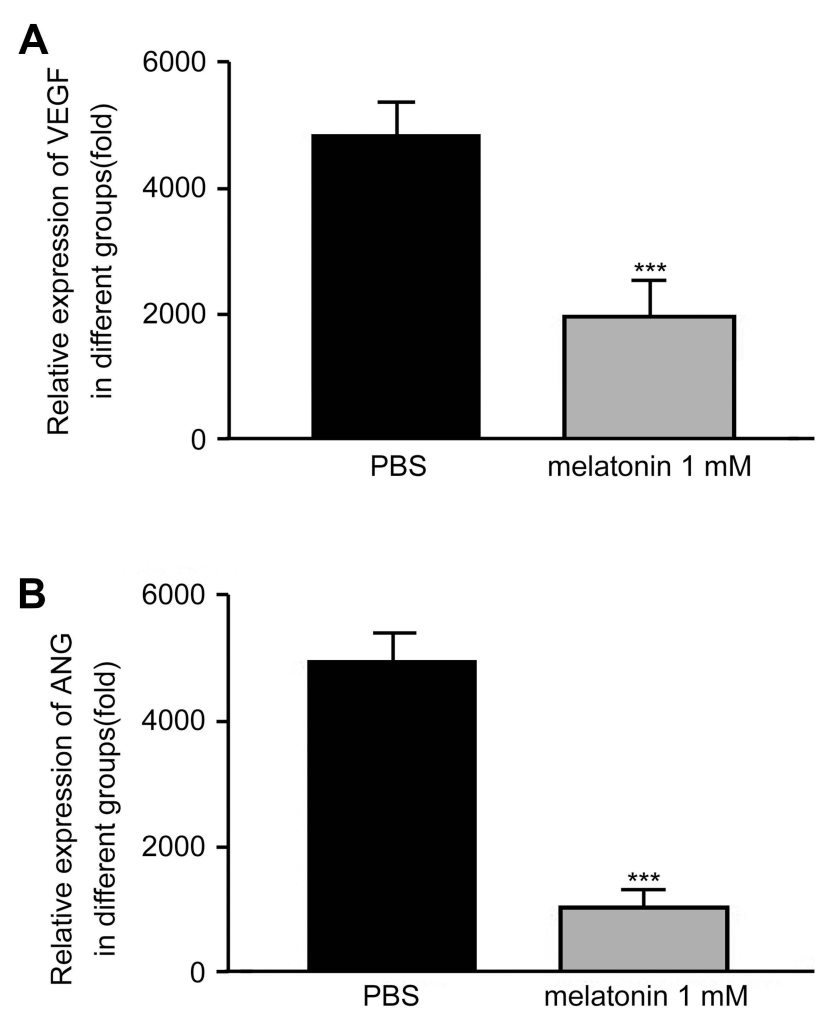

Figure 3 VEGF and angiogenin levels are measured in the supernatant of the chick embryo tumor xenograft model using ELISA. (A) VEGF level; (B) Angiogenin level. Data are expressed as mean \pm standard error of the mean of three independent experiments. $* * * P<0.05$ vs controls.

Abbreviations: VEGF, vascular endothelial growth factor, Ang, angiogenin.

in vitro proliferation of SGC-7901 cells at a concentration-dependent manner.

Angiogenesis has shown an important role in tumor development and metastasis, which is mediated by the balance between pro-angiogenic and anti-angiogenic factors. ${ }^{29}$ Angiogenesis is a multifactorial multi-step process, and blockade of any process is effective to prevent tumor angiogenesis. $^{30}$ Inhibition of angiogenesis is therefore accepted as a new target for antitumor therapy. ${ }^{16}$ Autocrine VEGF signaling is present in cancers, and autocrine VEGF/ VEGFR contributes to cancer proliferation, apoptosis and metastasis. ${ }^{31,32}$ VEGF has been identified as a key mediator of angiogenesis in cancers, and inhibition of VEGF signaling is effective to suppress the development of new blood vessels and tumor growth. ${ }^{32}$ Angiogenin, a member of the RNase superfamily, is a potent stimulator of angiogenesis, ${ }^{33}$ has been detected to be overexpressed and associated with poor clinical outcomes in multiple human cancers. ${ }^{34-36}$

In this study, the chicken embryos were inoculated with SGC-7901 cells from the 5th day after incubation, and MLT administered since the 7th day, with the day of grouping defined as Day 0. Treatment with MLT at $1 \mathrm{mM}$ was found to markedly reduce the volume and weight of tumors bearing the allantois of chicken embryos, and ELISA showed that treatment with MLT at $1 \mathrm{mM}$ significantly suppressed VEGF and angiopoietin production in chick embryo tumor xenograft models with SGC-7901 cells $(P=0.023)$, suggesting that MLT may suppress gastric cancer growth through inhibiting angiogenesis.

\section{Conclusions}

In summary, the results of the present study demonstrate that MLT inhibits gastric cancer growth in vitro at a concentration-dependent manner, and suppresses angiogenesis of the chick embryo tumor xenograft model with SGC-7901 cells through inhibiting VEGF and angiogenin secretion. Further studies are needed to investigate the therapeutic potential of MLT for gastric cancer as compared to drugs clinically approved.

\section{Acknowledgments}

The authors would like to thank the anonymous reviewers for their kind comments on the manuscript. This study was supported by the Young and Middle-aged Key Personnel Training Program of Fujian Provincial Health and Family Planning Commission (grant no. 2016-ZQN-51).

\section{Disclosure}

The authors declare no conflicts of interest.

\section{References}

1. Smyth EC, Nilsson M, Grabsch HI, van Grieken NC, Lordick F. Gastric cancer. Lancet. 2020;396(10251):635-648. doi:10.1016/ S0140-6736(20)31288-5

2. IARC. Global Cancer Observatory: cancer today. Lyon: International Agency for Research on Cancer; 2020. Available from: https://gco. iarc.fr/today. Accessed November 18, 2021.

3. Fitzmaurice C, Abate D; Global Burden of Disease Cancer Collaboration. et al. Global, regional, and national cancer incidence, mortality, years of life lost, years lived with disability, and disability-adjusted life-years for 29 cancer groups, 1990 to 2017: a systematic analysis for the global burden of disease study. JAMA Oncol. 2019;5(12):1749-1768. doi:10.1001/jamaoncol.2019.2996.

4. Venerito M, Link A, Rokkas T, Malfertheiner P. Gastric cancer clinical and epidemiological aspects. Helicobacter. 2016;21(Suppl 1):39-44. doi:10.1111/hel.12339

5. Tan YK, Fielding JW. Early diagnosis of early gastric cancer. Eur J Gastroenterol Hepatol. 2006;18(8):821-829. doi:10.1097/00042737200608000-00004

6. Digklia A, Wagner AD. Advanced gastric cancer: current treatment landscape and future perspectives. World J Gastroenterol. 2016;22 (8):2403-2414. doi:10.3748/wjg.v22.i8.2403

7. Sexton RE, Al Hallak MN, Diab M, Azmi AS. Gastric cancer: a comprehensive review of current and future treatment strategies. Cancer Metastasis Rev. 2020;39(4):1179-1203. doi:10.1007/s10555020-09925-3 
8. Abadi AJ, Zarrabi A, Hashemi F, et al. The role of SOX family transcription factors in gastric cancer. Int $J$ Biol Macromol. 2021;180:608-624. doi:10.1016/j.ijbiomac.2021.02.202

9. Ashrafizadeh M, Zarrabi A, Orouei S, et al. STAT3 pathway in gastric cancer: signaling, therapeutic targeting and future prospects. Biology. 2020;9(6):126.

10. Ashrafizadeh M, Rafiei H, Mohammadinejad R, Farkhondeh T, Samarghandian S. Anti-tumor activity of resveratrol against gastric cancer: a review of recent advances with an emphasis on molecular pathways. Cancer Cell Int. 2021;21(1):66. doi:10.1186/s12935-02101773-7

11. Ashrafizadeh M, Rafiei H, Mohammadinejad R, Farkhondeh T, Samarghandian S. Wnt-regulating microRNAs role in gastric cancer malignancy. Life Sci. 2020;250:117547. doi:10.1016/j.lfs.2020.117547

12. Griffioen AW, Dudley AC. Angiogenesis: a year in review. Angiogenesis. 2021;24(2):195-196. doi:10.1007/s10456-021-09798-2

13. Moriya J, Minamino T. Angiogenesis, cancer, and vascular aging. Front Cardiovasc Med. 2017;4:65. doi:10.3389/fcvm.2017.00065

14. Viallard C, Larrivée B. Tumor angiogenesis and vascular normalization: alternative therapeutic targets. Angiogenesis. 2017;20 (4):409-426. doi:10.1007/s10456-017-9562-9

15. Nienhüser H, Schmidt T. Angiogenesis and anti-Angiogenic therapy in gastric cancer. Int $J$ Mol Sci. 2017;19(1):43. doi:10.3390/ ijms 19010043

16. Li T, Kang G, Wang T, Huang H. Tumor angiogenesis and anti-angiogenic gene therapy for cancer. Oncol Lett. 2018;16 (1):687-702. doi:10.3892/o1.2018.8733

17. Amaral FGD, Cipolla-Neto J. A brief review about melatonin, a pineal hormone. Arch Endocrinol Metab. 2018;62(4):472-479. doi:10.20945/2359-3997000000066

18. Claustrat B, Leston J. Melatonin: physiological effects in humans. Neurochirurgie. 2015;61(2-3):77-84. doi:10.1016/j.neuchi.2015.03.002

19. Tordjman S, Chokron S, Delorme R, et al. Melatonin: pharmacology, functions and therapeutic benefits. Curr Neuropharmacol. 2017;15 (3):434-443. doi:10.2174/1570159X14666161228122115

20. Reiter RJ, Tan DX, Galano A. Melatonin: exceeding expectations. Physiology. 2014;29(5):325-333. doi:10.1152/physiol.00011.2014

21. Reiter RJ, Rosales-Corral SA, Tan DX, et al. Melatonin, a full service anti-cancer agent: inhibition of initiation, progression and metastasis. Int J Mol Sci. 2017;18(4):843. doi:10.3390/ijms18040843

22. Liu $\mathrm{H}, \mathrm{Zhu} \mathrm{Y}, \mathrm{Zhu} \mathrm{H}$, et al. Role of transforming growth factor $\beta 1$ in the inhibition of gastric cancer cell proliferation by melatonin in vitro and in vivo. Oncol Rep. 2019;42(2):753-762. doi:10.3892/or.2019.7190

23. Song J, Ma SJ, Luo JH, et al. Melatonin induces the apoptosis and inhibits the proliferation of human gastric cancer cells via blockade of the AKT/MDM2 pathway. Oncol Rep. 2018;39(4):1975-1983. doi:10.3892/or.2018.6282
24. Wang RX, Liu H, Xu L, Zhang H, Zhou RX. Involvement of nuclear receptor RZR/ROR $\gamma$ in melatonin-induced HIF-1 $\alpha$ inactivation in SGC-7901 human gastric cancer cells. Oncol Rep. 2015;34 (5):2541-2546. doi:10.3892/or.2015.4238

25. Wang RX, Liu H, Xu L, Zhang H, Zhou RX. Melatonin downregulates nuclear receptor $\mathrm{RZR} / \mathrm{ROR} \gamma$ expression causing growthinhibitory and anti-angiogenesis activity in human gastric cancer cells in vitro and in vivo. Oncol Lett. 2016;12(2):897-903. doi:10.3892/ol.2016.4729

26. Cheng J, Yang HL, Gu CJ, et al. Melatonin restricts the viability and angiogenesis of vascular endothelial cells by suppressing HIF-1 $\alpha /$ ROS/VEGF. Int J Mol Med. 2019;43(2):945-955. doi:10.3892/ ijmm.2018.4021

27. Peng W, Lei M, Zhang J, Zhang Y. The protective effect of melatonin on the in vitro development of yak embryos against hydrogen peroxide-induced oxidative injury. Zygote. 2019;27(3):118-125. doi:10.1017/S0967199418000412

28. Ma Q, Reiter RJ, Chen Y. Role of melatonin in controlling angiogenesis under physiological and pathological conditions. Angiogenesis. 2020;23(2):91-104. doi:10.1007/s10456-019-09689-7

29. Lugano R, Ramachandran M, Dimberg A. Tumor angiogenesis: causes, consequences, challenges and opportunities. Cell Mol Life Sci. 2020;77(9):1745-1770. doi:10.1007/s00018-019-03351-7

30. Li J, Li C, Yuan W, et al. Targeted temperature management suppresses hypoxia-inducible factor- $1 \alpha$ and vascular endothelial growth factor expression in a pig model of cardiac arrest. Neurocrit Care. 2021;35(2):379-388. doi:10.1007/s12028-020-01166-0

31. Lin Y, Zhai E, Liao B, et al. Autocrine VEGF signaling promotes cell proliferation through a PLC-dependent pathway and modulates Apatinib treatment efficacy in gastric cancer. Oncotarget. 2017;8 (7):11990-12002. doi:10.18632/oncotarget.14467

32. Carmeliet P. VEGF as a key mediator of angiogenesis in cancer. Oncology. 2005;69(Suppl 3):4-10. doi:10.1159/000088478

33. Tello-Montoliu A, Patel JV, Lip GY. Angiogenin: a review of the pathophysiology and potential clinical applications. J Thromb Haemost. 2006;4(9):1864-1874. doi:10.1111/j.1538-7836.2006.01 995.x

34. Sheng J, Xu Z. Three decades of research on angiogenin: a review and perspective. Acta Biochim Biophys Sin. 2016;48(5):399-410. doi:10.1093/abbs/gmv131

35. Pavlov N, Badet J. Angiogenin: involvement in angiogenesis and tumour growth. Bull Cancer. 2001;88(8):725-732.

36. Miyake M, Goodison S, Lawton A, Gomes-Giacoia E, Rosser CJ. Angiogenin promotes tumoral growth and angiogenesis by regulating matrix metallopeptidase-2 expression via the ERK1/2 pathway. Oncogene. 2015;34(7):890-901. doi:10.1038/onc.2014.2

\section{Publish your work in this journal}

Cancer Management and Research is an international, peer-reviewed open access journal focusing on cancer research and the optimal use of preventative and integrated treatment interventions to achieve improved outcomes, enhanced survival and quality of life for the cancer patient.
The manuscript management system is completely online and includes a very quick and fair peer-review system, which is all easy to use. Visit http://www.dovepress.com/testimonials.php to read real quotes from published authors. 